\title{
On the existence of minimizing domains for some shape optimization problems
}

\author{
Giuseppe Buttazzo *
}

\begin{abstract}
We describe some shape optimization problems of the form $\min \{\Phi(A): A \in \mathcal{A}\}$ and we show that, even if in general one could expect only relaxed solutions, in some particular cases the minimizing domains exist.
\end{abstract}

\section{Introduction}

We can describe a shape optimization problem as a minimum problem of the form

$$
\min \{\Phi(A): A \in \mathcal{A}\}
$$

where $\Phi$ is a cost functional which has to be minimized over a class $\mathcal{A}$ of admissible domains. Problems of this kind arise in various questions of mechanics and structural engineering, where the shape optimization problem has the form of an optimal control problem, that is the cost $\Phi$ is of the form

$$
\Phi(A)=\int_{\Omega} \varphi\left(x, u_{A}\right) d x
$$

being $u_{A}$ the solution of some partial differential equation in $A$. Volume constraints like $|A| \leq$ $k$, or penalization terms like $\int_{A} j(x) d x$ seems also raisonable conditions to be considered.

In this paper we want to present a survey of some recent results obtained in the cases when the partial differential state equation is of elliptic type, with Dirichlet boundary conditions. Other cases, with Neumann or transmission boundary conditions, have been widely studied in the literature (we refer for instance to the papers by Kohn and Strang [54], and by Murat and Tartar [61]) ; without any claim to be exhaustive we list in the references some of the many papers appeared.

A section will be also devoted to the case of determination of the optimal distribution density of material, with respect to the energy cost functional, when dealing with concentrated loads; this could be seen as an optimal sizing problem, and one should expect that the optimal density is, in some situations, a measure possibly not absolutely continuous with respect to the Lebesgue measure. The question of how to write the energy of the system when the material density may concentrate to a measure is then discussed.

The plan of the paper is the following.

1. Introduction

2. General formulation of an optimal control problem and its relaxation.

3. Shape optimization problems with Dirichlet boundary conditions.

*Dipartimento di Matematica - Università di Pisa, Via Buonarroti, 2 - 56127 PISA (ITALY), e-mail : buttazzo@sabsns.sns.it, buttazzo@dm.unipi.it 
4. Existence of classical domains as minimizers under geometrical constraints.

5. Existence of classical domains as minimizers under monotonicity assumptions on the cost functional.

6. Minimum problems for functions of eigenvalues.

7. Optimal distribution densities for concentrated loads.

\section{General formulation of an optimal control problem and its relaxation}

A general formulation of an optimal control problem requires an abstract framework, large enough to include cases, as the shape optimization one, when no topological structures on the set of admissible controls are a priori given. We consider:

$U$ the space of states, we assume it is a topological space ;

$\mathcal{A}$ the set of controls, no topology on $\mathcal{A}$ is a priori considered ;

$J$ the cost function defined on $\mathcal{A} \times U$;

$G$ a function defined on $\mathcal{A} \times U$ which provides the state equation as a minimization problem of the form

$$
u \in \operatorname{argmin} G(A, \cdot)
$$

we may call $G$ the state functional. When for every control $A$ the minimization of the state functional provides a unique minimizer, we denote this minimizer by $u_{A}$, to stress its dependence on $A$.

Therefore, the optimal control problem can be written as

$$
\min \{J(A, u): A \in \mathcal{A}, u \in U, u \in \operatorname{argmin} G(A, \cdot)\} .
$$

Note that here the cost function $J$ may depend both on $A$ and $u_{A}$. Problems of this form are very common in a lot of applications to structural engineering, where $A$ can be the design of a mechanical structure, $u$ its displacement, and the cost $J$ may take into account the weight of the structure and some desired performances given in terms of the displacement $u$.

In several situations problem (1) does not admit any solution, and a "relaxed" problem

$$
\min \{\hat{J}(\hat{A}, u): \hat{A} \in \hat{\mathcal{A}}, u \in U, u \in \operatorname{argmin} \hat{G}(\hat{A}, \cdot)\}
$$

is introduced, where $\hat{\mathcal{A}}$ is the class of "relaxed" domains, $\hat{J}$ the "relaxed" cost functional, and $\hat{G}$ the "relaxed" state functional, suitably defined. In order to achieve this goal, we make the following assumptions:

the space $U$ of states is a separable metric space ;

for every $A \in \mathcal{A}$ the function $G(A, \cdot)$ is proper and lower semicontinuous on $U$;

there exists a lower semicontinuous function $\psi: U \rightarrow \overline{\mathbf{R}}$ which is coercive, in the sense

that for every $t \in \mathbf{R}$ the sublevel set $\{\psi \leq t\}$ is compact in $U$, such that

$$
G(A, u) \geq \psi(u) \quad \forall(A, u) \in \mathcal{A} \times U
$$

ESAIM: PROC., VOL. 3, 1998, 51-64 
Under the assumptions above (see for instance Dal Maso [44], Chapter 10) the class $S_{\psi}(U)$ of all lower semicontinuous functions from $U$ into $\overline{\mathbf{R}}$ minorized by $\psi$, is a compact metric space once it is endowed by the $\Gamma$-convergence topology ; we denote by $\delta_{\Gamma}$ the associated distance. Therefore, if we denote by $\mathcal{G}: \mathcal{A} \rightarrow S_{\psi}(U)$ the mapping given by $\mathcal{G}(A)=G(A, \cdot)$ and we assume in addition

the mapping $\mathcal{G}$ is one-to-one,

setting

$$
d(A, B)=\delta_{\Gamma}(\mathcal{G}(A), \mathcal{G}(B)),
$$

we get that $d$ is a distance on $\mathcal{A}$ and $\mathcal{G}$ is an isometry between $(\mathcal{A}, d)$ and $\left(S_{\psi}(U), \delta_{\Gamma}\right)$. We denote by $\gamma$ the convergence associated to the distance $d$; therefore

$$
A_{h} \rightarrow A \text { in the } \gamma \text {-convergence on } \mathcal{A} \Longleftrightarrow \Gamma \lim _{h \rightarrow+\infty} G\left(A_{h}, \cdot\right)=G(A, \cdot)
$$

Remark 2.1. Since the $\Gamma$-convergence implies the convergence of minima and of minimum points (see for instance Dal Maso [44]), the $\gamma$-convergence topologizes $\mathcal{A}$ in the most natural way with respect to the minimum problem (1).

We are now in a position to introduce the relaxed controls and to define the relaxed problem associated to $(1)$. We define by $(\hat{\mathcal{A}}, \hat{d})$ the completion of the metric space $(\mathcal{A}, d)$ and by $\hat{\mathcal{G}}: \hat{\mathcal{A}} \rightarrow \overline{\mathbf{R}}$ the unique isometry which extends $\mathcal{G}$; more precisely we set

$$
\hat{\mathcal{G}}(\hat{A})=\Gamma \lim _{h \rightarrow+\infty} \mathcal{G}\left(A_{h}\right)
$$

where $\left\{A_{h}\right\}_{h}$ is any sequence converging to $\hat{A}$ in $(\hat{\mathcal{A}}, \hat{d})$. In other words, if $\hat{G}: \hat{\mathcal{A}} \times U \rightarrow \overline{\mathbf{R}}$ is defined by $\hat{G}(\hat{A}, \cdot)=\hat{\mathcal{G}}(\hat{A})$, we can extend the $\gamma$-convergence to all of $\hat{\mathcal{A}}$ and we have

$$
\hat{A}_{h} \rightarrow \hat{A} \text { in the } \gamma \text {-convergence on }(\hat{\mathcal{A}}, \hat{d}) \quad \Longleftrightarrow \quad \Gamma \lim _{h \rightarrow+\infty} \hat{G}\left(\hat{A}_{h}, \cdot\right)=\hat{G}(\hat{A}, \cdot) \text {. }
$$

The compactness of the $\Gamma$-convergence on $S_{\psi}(U)$ implies that $(\hat{\mathcal{A}}, \hat{d})$ is a compact metric space. The elements of $\hat{\mathcal{A}}$ are called relaxed controls and $\hat{G}$ is the relaxed state functional. In order to identify the relaxed control problem associated to (1) we assume

for every $\hat{A} \in \hat{\mathcal{A}}$ the minimum point of $\hat{G}(\hat{A}, \cdot)$ on $U$ is unique ;

there exists a function $\Phi: \mathcal{A} \rightarrow \mathbf{R}$ bounded on $d$-bounded sets, and a function

$$
\begin{aligned}
& \omega: U \times U \rightarrow \mathbf{R} \text { with } \lim _{v \rightarrow u} \omega(u, v)=0 \text {, for all } u \in U, \text { such that } \\
&|J(A, u)-J(A, v)| \leq \Phi(A) \omega(u, v) \quad \forall A \in \mathcal{A}, \forall u, v \in U .
\end{aligned}
$$

Theorem 2.2. (see Belloni, Buttazzo, Freddi [5]) Under assumptions (2)-(7) the relaxed problem associated to (1) is given by

$$
\min \{\hat{J}(\hat{A}, u): \hat{A} \in \hat{\mathcal{A}}, u \in U, u \in \operatorname{argmin} \hat{G}(\hat{A}, \cdot)\}
$$


where $\hat{J}: \hat{\mathcal{A}} \times U \rightarrow \overline{\mathbf{R}}$ is the relaxed cost functional

$$
\hat{J}(\hat{A}, u)=\inf \left\{\liminf _{h \rightarrow+\infty} J\left(A_{h}, u\right): A_{h} \rightarrow \hat{A} \text { in } \hat{\mathcal{A}}\right\} .
$$

Problem (8) admits always a solution, the minimizing sequences of (1) $\gamma$-converge (up to subsequences) to minimum points of (8), and the infimum of (1) coincides with the minimum of (8).

Remark 2.3. When the cost functional $J$ does not depend on the control variable $A$, we do not need to perform any relaxation in the cost ; indeed problem (8) has the simpler form

$$
\min \{J(u): \hat{A} \in \hat{\mathcal{A}}, u \in U, u \in \operatorname{argmin} \hat{G}(\hat{A}, \cdot)\} .
$$

\section{Shape optimization problems with Dirichlet boundary conditions}

As an application of the general framework illustrated in the previous section we consider here a class of shape optimization problems governed by an elliptic equation with Dirichlet conditions on the free boundary, and we identify their relaxed formulation. In the following $\Omega$ will be a given bounded open subset of $\mathbf{R}^{n}(n \geq 2)$ with a Lipschitz boundary, and $f$ will be a given element of $H^{-1}(\Omega)$. For the sake of simplicity, as a model operator we shall take the Laplace operator $-\Delta$, but much could be extended to a more general class of second order elliptic operators. With the notation of Section 2 we have

$U$ is the space $H_{0}^{1}(\Omega)$ endowed with the strong $L^{2}(\Omega)$ topology ;

$\mathcal{A}$ is the class of all open subsets $A$ of $\Omega$;

$G$ is the state functional

$$
G(A, u)= \begin{cases}\int_{\Omega}|D u|^{2} d x-2\langle f, u\rangle & \text { if } u \in H_{0}^{1}(A) \\ +\infty & \text { otherwise }\end{cases}
$$

which provides, via minimization, the elliptic state equation

$$
\begin{cases}-\Delta u=f & \text { in } A \\ u=0 & \text { on } \Omega \backslash A ;\end{cases}
$$

$J$ is an integral cost functional of the form

$$
J(u)=\int_{\Omega} j(x, u) d x
$$

in which there is no explicit dependence on the control variable $A$, or more generally

$$
J(A, u)=\int_{A} j(x, u) d x
$$

where $j(x, s)$ is a Carathéodory integrand satisfying

$$
|j(x, s)| \leq a(x)+b|s|^{2}
$$

for suitable $a \in L^{1}(\Omega)$ and $b \geq 0$. 
In this case the characterization of the space $\hat{\mathcal{A}}$ of relaxed controls has been obtained by Dal Maso and Mosco in [48], where it is proved that $\hat{\mathcal{A}}$ coincides with the class $\mathcal{M}_{0}$ of all Borel measures $\mu: \mathcal{B}(\Omega) \rightarrow[0,+\infty]$ which vanish on all sets of capacity zero. Accordingly, the relaxed cost $\hat{G}$ is then characterized as the functional given by

$$
\hat{G}(\mu, u)=\int_{\Omega}|D u|^{2} d x-2\langle f, u\rangle+\int_{\Omega} u^{2} d \mu
$$

so that the relaxed state equation $u \in \operatorname{argmin} \hat{G}(\mu, \cdot)$ can be written as

$$
\left\{\begin{array}{l}
-\Delta u+\mu u=f \\
u \in H_{0}^{1}(\Omega) \cap L_{\mu}^{2}(\Omega) .
\end{array} \quad \text { in } \Omega\right.
$$

In this framework we shall call the elements of $\mathcal{M}_{0}$ "relaxed domains".

Remark 3.1. Since $u \in H_{0}^{1}(\Omega)$, the value $u(x)$ is defined almost everywhere in capacity, hence the integral $\int_{\Omega} u^{2} d \mu$ is well defined. Moreover, it is easy to see that the "classical domains" $A \in \mathcal{A}$ can be written as particolar measures of the class $\mathcal{M}_{0}$; indeed, if

$$
\mu(B)=\infty_{\Omega \backslash A}(B)= \begin{cases}0 & \text { if } \operatorname{cap}(A \backslash B)=0 \\ +\infty & \text { otherwise }\end{cases}
$$

we have $\hat{G}(\mu, \cdot)=G(A, \cdot)$ and equation (11) reduces to the original equation

$$
\begin{cases}-\Delta u=f & \text { in } A \\ u=0 & \text { on } \Omega \backslash A\end{cases}
$$

It remains to characterize the relaxed cost functional $\hat{J}$. If $J$ is of the form (9) without any explicit dependence on $A$, then, as we already mentioned in Remark 2.3 , we have $\hat{J}=J$ and so the relaxed shape optimization problem reads

$$
\min \left\{\int_{\Omega} j(x, u) d x: \mu \in \mathcal{M}_{0}, u \in H_{0}^{1}(\omega),-\Delta u+\mu u=f\right\} .
$$

When $J$ is of the form (10) Buttazzo, Dal Maso, Garroni and Malusa [28] obtained the following expression for $\hat{J}$ :

$$
\hat{J}(\mu, u)=\int_{A_{\mu}} j(x, u) d x-\int_{\Omega \backslash A_{\mu}} j^{-}(x, 0) d x
$$

being, roughly speaking, $A_{\mu}$ the set where $\mu$ "is finite". More precisely, if for every $\mu \in \mathcal{M}_{0}$ we denote by $w_{\mu}$ the solution of

$$
\left\{\begin{array}{l}
-\Delta w+\mu w=1 \\
w \in H_{0}^{1}(\Omega) \cap L_{\mu}^{2}(\Omega)
\end{array}\right.
$$

then $A_{\mu}$ coincides with the set $\left\{w_{\mu}>0\right\}$.

Remark 3.2. In a similar way (see [28]) we can treat cost functionals of the form

$$
J(A, u)=\int_{A} j(x, u) d \lambda
$$

where $\lambda$ is a given finite measure of $\mathcal{M}_{0}$ and $j(x, s)$ is an integrand such that

(i) for every $u \in H_{0}^{1}(\Omega)$ the function $j(x, u(x))$ belongs to $L_{\lambda}^{1}(\Omega)$; 
(ii) the map $u \mapsto \int_{\Omega} j(x, u) d \lambda$ is sequentially weakly continuous in $H_{0}^{1}(\Omega)$.

In this case we get

$$
\hat{J}(\mu, u)=\int_{A_{\mu}} j(x, u) d \lambda-\int_{\Omega \backslash A_{\mu}} j^{-}(x, 0) d \lambda .
$$

The case when a constraint of the form $|A| \in T$ is present in the original shape optimization problem, can be also treated (see [28]), even if in a slightly more complicated way; here $T$ is any subset of the interval $[0,|\Omega|]$ and $|\cdot|$ denotes the Lebesgue measure (or the measure $\lambda$ if $J$ is given by (12)). Then we obtain for a cost functional $J$ of the form (9) the expression

$$
\hat{J}(\mu, u)= \begin{cases}\int_{\Omega} j(x, u) d x & \text { if }\left|A_{\mu}\right| \leq \sup T \\ +\infty & \text { otherwise }\end{cases}
$$

and for a cost functional $J$ of the form (10) the expression

$$
\hat{J}(\mu, u)=\int_{A_{\mu}} j(x, u) d x+\inf \left\{\int_{B \backslash A_{\mu}} j(x, 0) d x: A_{\mu} \subset B,|B| \in T\right\} .
$$

The following argument, by Chipot and Dal Maso [41] shows that in some cases a relaxed shape optimization problem with Dirichlet conditions on the free boundary can be reduced to a minimization problem over a convex set, and therefore the uniqueness of the relaxed solution can be obtained in several situations. Indeed, the relaxed equation

$$
\left\{\begin{array}{l}
-\Delta u+\mu u=f \\
u \in H_{0}^{1}(\Omega) \cap L_{\mu}^{2}(\Omega),
\end{array} \quad \text { in } \Omega\right.
$$

in case of a nonnegative right hand side $f$, by the maximum principle, provides a nonnegative solution $u$. Therefore, the set of all $u \in H_{0}^{1}(\Omega)$ such that there exists $\mu \in \mathcal{M}_{0}$ satisfying the equation above, turns out to be equal to the closed convex subset of $H_{0}^{1}(\Omega)$

$$
\mathcal{K}=\left\{u \in H_{0}^{1}(\Omega): \Delta u+f \geq 0\right\} .
$$

Moreover, the mapping which associates to every measure $\mu \in \mathcal{M}_{0}$ the solution $u_{\mu}$ of the relaxed state equation is one-to-one because it can be shown that $\mu$ can be deduced by $u$ through the equality

$$
\mu=\frac{\Delta u+f}{u}
$$

where it is intended that $\mu=+\infty$ when $u=0$. In this way, the shape optimization problem

$$
\min \left\{J(u): u \in H_{0}^{1}(\Omega), \mu \in \mathcal{M}_{0},-\Delta u+\mu u=f\right\}
$$

becomes equivalent to the minimization problem

$$
\min \{J(u): u \in \mathcal{K}\} .
$$

Since $\mathcal{K}$ is closed and convex, if the cost functional $J$ is strictly convex this last problem has a unique solution $u_{\text {opt }} \in \mathcal{K}$, and so the relaxed shape optimization (14) has the unique solution

$$
\mu_{o p t}=\left(\Delta u_{o p t}+f\right) / u_{o p t}
$$

Example 3.3. Taking the admissible class $\mathcal{A}$ equal to the family of all subdomains of $\Omega$, it is possible to show (see [26]) that for a general choice of the data $f$ and $g$ the shape optimization problem

$$
\min \left\{\int_{\Omega}|u-g(x)|^{2} d x: u \in H_{0}^{1}(\Omega), A \in \mathcal{A},-\Delta u=f \text { in } A\right\}
$$

ESAIM: Proc., VOL. 3, 1998, 51-64 
has no solutions, so that the relaxed problem

$$
\min \left\{\int_{\Omega}|u-g(x)|^{2} d x: u \in H_{0}^{1}(\Omega), \mu \in \mathcal{M}_{0},-\Delta u+\mu u=f\right\}
$$

must be considered. According to what said just above, if $f \geq 0$ we are in the position to write the relaxed problem in the form

$$
\min \left\{\int_{\Omega}|u-g(x)|^{2} d x: u \in \mathcal{K}\right\}
$$

and this problem has a unique solution $u$ (and hence $\mu$ by (13)), due to the strict convexity of the functional $\int_{\Omega}|u-g(x)|^{2} d x$.

\section{Existence of classical domains as minimizers under geometrical constraints}

As we have seen in Example 3.3, in general one should expect only relaxed solutions for a shape optimization problem. In order to "force" the existence of a classical nonrelaxed minimizing domain, two approaches are possible:

(i) add more constraints on the class $\mathcal{A}$ of admissible domains ;

(ii) consider more restricted classes of cost functionals.

In this section we consider the approach (i), which is more studied in the literature ; for instance, if we take the minimum on the class $\mathcal{A}_{k}$ of all domains which satisfy a Lipschitz condition with a uniform constant $k$, then the existence of a domain solution in $\mathcal{A}_{k}$ can be obtained for a large class of cost functionals. A condition weaker than the $k$-Lipschitz one and which still implies the existence of a classical domain as a minimizer, is the so called "exterior cone condition" which consists in assuming that the admissible domains $A$ have, for each point $x$ at their boundary, a cone with uniform height and opening, centered at $x$ and exterior to $A$. We refer to the book of Pironneau [63] and to some papers by Chenais [38], [39], [40] for further details on the exterior cone conditions for elliptic equations.

More recently, a result due to Bucur and Zolesio [15], [16], [17] generalizes the results above, and gives the $\gamma$-compactness of the class of admissible domains (hence the existence of a classical domain as a minimizer) under a sole capacitary condition of the form:

there exist $c>0$ and $r_{0}>0$ such that

$$
\forall x \in \partial A, \quad \forall r<r_{0} \quad \frac{\operatorname{cap}(B(x, r) \backslash A, B(x, 2 r))}{\operatorname{cap}(B(x, r), B(x, 2 r))} \geq c,
$$

or more generally

there exists a function $w:(0,1) \times(0,1) \times \Omega \rightarrow \mathbf{R}^{+}$such that

i) $w$ is lower semicontinuous in the third variable;

ii) $\lim _{r \rightarrow 0} w(r, R, x)=+\infty$, locally uniformly on $x$;

iii) for every $x \in \partial A$ and every $0<r<R<1$

$$
\int_{r}^{R}\left(\frac{\operatorname{cap}(B(x, t) \backslash A, B(x, 2 t))}{\operatorname{cap}(B(x, t), B(x, 2 t))}\right) \frac{d t}{t} \geq w(r, R, x) .
$$

Finally, still in the framework of the approach (i), we have to mention a result by Sverak [66] [67]: in the case of dimension $n=2$ the topological condition on the admissible domains 
the number of connected components of $\Omega \backslash A$ is less or equal to $k$

still implies the $\gamma$-compactness and by consequence the existence of a classical domain as a minimizer.

The results above have been recently generalized to the nonlinear case by Bucur and Trebeschi [14] who proved the following compactness result for shape optimization problems governed by the $p$-Laplace operator $-\Delta_{p}$ or more generally by a monotone operator of the form - div $(a(x, D u))$ "which behaves" like $-\Delta_{p}$. Correspondingly, the $p$-capacity $C_{p}$ and the $\gamma_{p}$-convergence are defined in a similar way.

Theorem 4.1. We have:

i) if $p>n$ then the whole class of open subsets of $\Omega$ is $\gamma_{p}$-compact, by the Sobolev embedding theorem ;

ii) if $1<p \leq n$ then the class $\mathcal{A}_{w}$ of all open subsets $A$ of $\Omega$ such that for all $x \in \partial A$ and all $0<r<R<1$

$$
\int_{r}^{R}\left(\frac{C_{p}(B(x, t) \backslash A, B(x, 2 t))}{C_{p}(B(x, t), B(x, 2 t))}\right)^{\frac{1}{p-1}} \frac{d t}{t} \geq w(r, R, x)
$$

is $\gamma_{p}$-compact, provided $w:(0,1) \times(0,1) \times \Omega \rightarrow \mathbf{R}^{+}$is a given function such that

(a) $w$ is lower semicontinuous in the third variable,

(b) $\lim _{r \rightarrow 0} w(r, R, x)=+\infty$, locally uniformly on $x$.

Moreover, if $n-1<p \leq n$ a result similar to the Sverak one holds:

(c) the class of all open subsets of $\Omega$ such that the number of connected components of $\Omega \backslash A$ is less or equal to $k$ is $\gamma_{p}$-compact.

\section{Existence of classical domains as minimizers under monotonicity assumptions on the cost functional}

The approach of considering more restricted classes of cost functionals by leaving the admissible domains of $\mathcal{A}$ without any geometrical constraint, is more recent: the following result has been proved by Buttazzo and Dal Maso [27]. Here by $\Omega$ we denote a given bounded open subset of $\mathbf{R}^{n}$, and by $\mathcal{A}$ the class of all quasi open subsets of $\Omega$, that is of all sets $A$ such that for every $\varepsilon>0$ there exists an open subset $A_{\varepsilon}$ of $\Omega$ with $\operatorname{cap}\left(A_{\varepsilon} \Delta A\right)<\varepsilon$, where $\Delta$ is the symmetric difference of sets.

Theorem 5.1. Let $\Phi: \mathcal{A} \rightarrow \overline{\mathbf{R}}$ be a mapping satisfying the following assumptions:

(i) $\Phi$ is lower semicontinuous with respect to the $\gamma$-convergence ;

(ii) $\Phi$ is decreasing with respect to the set inclusion, i.e., $\Phi(A) \geq \Phi(B)$ whenever $A \subset B$. Then for every $c$ between 0 and $|\Omega|$ the minimum

$$
\min \{\Phi(A): A \in \mathcal{A},|A|=c\}
$$

is achieved.

Remark 5.2. The lower semicontinuity assumption (i) in Theorem 5.1 above is quite involved, but it is fulfilled in a large number of situations. For instance, if

$$
\Phi(A)=\int_{\Omega} j\left(x, u_{A}\right) d x
$$

ESAIM: Proc., VOL. 3, 1998, 51-64 
where $u_{A}$ is the solution of

$$
\begin{cases}-\Delta u=f & \text { in } A \\ u=0 & \text { on } \Omega \backslash A,\end{cases}
$$

it is easy to see that $\Phi$ is actually continuous with respect to the $\gamma$-convergence under a sole growth condition on the integrand $j$. Analogously, it is possible to show (see Buttazzo and Dal Maso [27], Example 2.1) that every continuous function of the eigenvalues of the Laplace operator is $\gamma$-continuous ; this kind of functions will be considered in more details in the next section.

On the other hand, the monotonicity assumption (ii) is very severe, and must be considered as the one which actually "forces" the existence of a classical domain as a minimum instead

of a general measure of $\mathcal{M}_{0}$. In the case of integral functionals of the form (16) and equation (17) with right-hand side $f \geq 0$, by the maximum principle we obtain that $\Phi$ is decreasing with respect to the set inclusion whenever the integrand $j(x, s)$ is decreasing with respect to $s$. For instance, in general the quadratic functional of Example 3.3 is not decreasing with respect to the set inclusion, and in fact we have to expect in this case only a relaxed solution $\mu$ for the shape optimization problem.

Remark 5.3. Theorem 5.1 above shows that the monotonicity assumption on the cost $\Phi$ rules out the possibility for the minimum point to be a measure, and the existence of an optimal quasi open domain follows. An interesting question would be to study conditions on the cost functional under which the optimal domains are actually open sets and the regularity of their boundary.

\section{Minimum problems for functions of eigenvalues}

By Theorem 5.1, using the fact that the eigenvalues of the Laplace operator with Dirichlet boundary conditions are decreasing with respect to the set inclusion, and taking into account Remark 5.2, we obtain immediately that for every fixed integer $N$ and $c \in[0, \Omega]$ all minimum problems of the form

$$
\min \left\{\varphi\left(\lambda_{1}(A), \ldots, \lambda_{N}(A)\right): A \in \mathcal{A},|A|=c\right\}
$$

have a classical domain solution provided $\mathcal{A}$ is the class of all quasi open subsets of $\Omega$, and $\varphi$ is a continuous (or more generally lower semicontinuous) function which is increasing in each variable.

However, in some cases the monotonicity assumption is not necessary for the existence of a classical optimal domain, even if we do not know a general condition weaker than monotonicity and which still imply the classical existence. We consider the case of a cost functional depending on the first two eigenvalues of the Laplace operator

$$
\Phi(A)=\varphi\left(\lambda_{1}(A), \lambda_{2}(A)\right)
$$

where $\varphi$ is a continuous (or lower semicontinuous) nonnegative function, and the corresponding minimum problem

$$
\min \left\{\Phi(A): A \in \mathcal{A}_{c}\right\}
$$

being $\mathcal{A}_{c}$ the class of all quasi open subsets of $\Omega$ having Lebesgue measure equal to $c$. By introducing the subset of $\mathbf{R}^{2}$

$$
E_{c}=\left\{(x, y) \in \mathbf{R}^{2}: x=\lambda_{1}(A), y=\lambda_{2}(A) \text { for some } A \in \mathcal{A}_{c}\right\}
$$


problem (20) can be written in the form

$$
\min \left\{\varphi(x, y):(x, y) \in E_{c}\right\} .
$$

It is therefore important to know if the set $E_{c}$ is closed in $\mathbf{R}^{2}$, since this would immediately imply an existence result for problem (20) under conditions much weaker than monotonicity (for instance the assumption $\varphi(x, y) \rightarrow+\infty$ as $|(x, y)| \rightarrow+\infty$ would suffice).

Some information about the set $E_{c}$ are available:

- if $(x, y) \in E_{c}$ then $x \geq 0, y \geq 0$, and $y \geq x$;

- if $(x, y) \in E_{c}$ then $x \geq \lambda_{1}(B)$ being $B$ the ball of measure $c$;

- if $(x, y) \in E_{c}$ then $y \geq \lambda_{1}(A)$ being $A$ the union of two disjoint ball of measure $c / 2$;

- if $(x, y) \in E_{c}$ then $(t x, t y) \in E_{c}$ for all $t \geq 1$;

- if $(x, y) \in E_{c}$ then $y \lambda_{1}(B) \leq x \lambda_{2}(B)$, by the result of Ashbaugh and Benguria [2].

The following result (see Bucur, Buttazzo and Figuereido [11]) concludes the analysis of the set $E_{c}$.

Theorem 6.1. For every constant $c \in[0,|\Omega|]$ the set $E_{c}$ is closed.

\section{Optimal distribution densities for concentrated loads}

In this section we consider the optimization problem for a material density once the loads are given and may possibly concentrate on sets of lower dimension. For simplicity we limit ourselves to the case of scalar functions: the general theory of the calculus of variations with respect to measures was recently introduced by Bouchittè, Buttazzo and Seppecher in [7], a first application to optimization will appear in [8], while the general framework suitable to include problems from elasticity is still in preparation. To set correctly the problem, consider a given signed measure $f$ on $\mathbf{R}^{n}$ with finite total variation and zero average ; in a stationary heat conduction model it represents the heat sources density which may possibly concentrate on sets of dimension lower than $n$. For every nonnegative measure $\mu$ on $\mathbf{R}^{n}$ (in the heat conduction model above it represents the conductivity density), we consider the total energy associated to a given smooth distribution temperature $u$

$$
E(\mu, u)=\frac{1}{2} \int|D u|^{2} d \mu-\langle f, u\rangle \quad\left(u \in \mathcal{D}\left(\mathbf{R}^{n}\right)\right)
$$

and we denote by $\mathcal{E}(\mu)$ its infimum

$$
\mathcal{E}(\mu)=\inf \left\{E(\mu, u): u \in \mathcal{D}\left(\mathbf{R}^{n}\right)\right\} .
$$

Of course, we may have $\mathcal{E}(\mu)=-\infty$ for some measures $\mu$; this happens for instance when the measure $f$ concentrates on sets of dimension smaller that $n-1$ and $\mu$ is the Lebesgue measure. These measures will be unrelevant for us because we consider the optimization problem

$$
\max \left\{\mathcal{E}(\mu): \mu \text { nonnegative measure, } \int d \mu=m\right\}
$$

where the total mass $m$ is a constant a priori given. The following result holds (see [8]).

Theorem 7.1. The shape optimization problem (22) admits a solution $\mu_{0}$.

Without entering into the details of the proof, we want to illustrate here some links between problem (22) and a kind of Monge-Kantorovich equation. One of the tools necessary

ESAIM: Proc., VoL. 3, 1998, 51-64 
to write rigorously this equation in the framework of measures as coefficients, is the notion of tangent space to a measure $T_{\mu}(x)$ and the related notion of tangential gradient $D_{\mu} u$. We refer to the paper [7] for the precise definitions, by remarking that in the case of a smooth manifold $S$ of dimension $k$ the related Hausdorff measure $\mathcal{H}^{k} L S$ provides the usual tangent space and the usual tangential gradients. The Monge-Kantorovich equation is then written as

$$
\left\{\begin{array}{l}
-\operatorname{div}\left(\mu D_{\mu} w\right)=f \\
w \in \operatorname{Lip}_{1}\left(\mathbf{R}^{n}\right), \quad\left|D_{\mu} w\right|=1 \mu \text {-a.e. }
\end{array}\right.
$$

where $\operatorname{Lip}_{1}\left(\mathbf{R}^{n}\right)$ is the class of all Lipschitz functions on $\mathbf{R}^{n}$ with constant 1 . Note that both $w$ and $\mu$ are unknown in equation (23).

Theorem 7.2. For every measure $f$ on $\mathbf{R}^{n}$ with finite total variation and zero average there exist a bounded nonnegative measure $\mu$ and a function $w \in \operatorname{Lip}_{1}\left(\mathbf{R}^{n}\right)$ satisfying the Monge-Kantorovich equation (23). Moreover, a measure $\mu$ solves the optimization problem (20) if and only if the rescaled measure $\mu|I(f)| / m$ solves the Monge-Kantorovich equation (23), where $I(f)$ is the quantity

$$
I(f)=\min \left\{-\langle f, \phi\rangle: \phi \in \operatorname{Lip}_{1}\left(\mathbf{R}^{n}\right)\right\} .
$$

\section{References}

[1] G. Allaire \& R.V. Kohn, Optimal design for minimum weight and compliance in plane stress using extremal microstructures, Europ. J. Mech. A/Solids, 12, (6), (1993), $839-878$.

[2] M.S. Ashbaugh \& R.D. Benguria, Proof of the Payne-Pólya-Weinberger conjecture, Bull. Amer. Math. Soc., 25, (1991), 19-29.

[3] H. AттоuсH, Variational convergence for functions and operators, Pitman, London, 1984.

[4] D. AzE \& G. ButTazzo, Some remarks on the optimal design of periodically reinforced structures, RAIRO Modél. Math. Anal. Numér., 23, (1989), 53-61.

[5] M. Belloni, G. Buttazzo \& L. Freddi, Completion by Gamma-convergence for optimal control problems, Ann. Fac. Sci. Toulouse Math., 2, (1993), 149-162.

[6] M. BendsøE, Optimal shape design as a material distribution problem, Struct. Optim., 1, (1989), 193-202.

[7] G. Bouchitte, G. Buttazzo \& P. Seppecher, Energies with respect to a measure and applications to low dimensional structures, Calc. Var., 5, (1997), 37-54.

[8] G. Bouchitte, G. Buttazzo \& P. Seppecher, Shape optimization solutions via Monge-Kantorovich equation, C. R. Acad. Sci. Paris, (to appear).

[9] A. Braides \& A. Malusa, Approximation of relaxed Dirichlet problems, Proceedings of Calculus of Variations, Homogenization and Continuum Mechanics, CIRM, MarseilleLuminy, 21-25 June 1993, World Scientific, Singapore, 1994.

[10] D. Bucur, Capacitary extension and two dimensional shape optimization, Preprint 93.24, Institut Non Linéaire, Nice, 1993.

[11] D. Bucur, G. Buttazzo \& I. Figuereido, On the attainable eigenvalues of the Laplace operator, Preprint Dipartimento di Matematica Università di Pisa, Pisa, 1997. 
[12] D. Bucur, G. Buttazzo \& A. Henrot, Existence results for some optimal partition problems, Preprint Dipartimento di Matematica Università di Pisa, Pisa, 1996.

[13] D. Bucur \& A. Henrot, Continuous Steiner symmetrization and Mosco convergence, (paper in preparation).

[14] D. Bucur \& P. Trebeschi, Shape optimization problems governed by nonlinear state equations, Preprint Dipartimento di Matematica Università di Pisa, Pisa, 1996.

[15] D. Bucur \& J.P. Zolesio, $N$-dimensional shape optimization under capacitary constraint, J. Diff. Eq., 123, (1995), 504-522.

[16] D. Bucur \& J.P. Zolesio, Wiener criterion and shape continuity for the Dirichlet problem, Preprint 94.32, Institut Non Linéaire, Nice, 1994.

[17] D. Bucur \& J.P. Zolesio, Flat cone condition and shape analysis, Control of Partial Differential Equations, G. Da Prato and L. Tubaro eds., Marcel Dekker Pubs., (to appear).

[18] G. Buttazzo, Thin insulating layers: the optimization point of view, Proceedings of Material Instabilities in Continuum Mechanics and Related Mathematical Problems, Edinburgh 1985-1986, edited by J. M. Ball, Oxford University Press, Oxford, (1988), 11-19.

[19] G. Buttazzo, An optimization problem for thin insulating layers around a conducting medium, Proceedings of the IFIP Conference Boundary Control and Boundary Variations, Nice 10-13 June 1986, edited by J. P. Zolésio, Lecture Notes in Control Inform. 100, Springer-Verlag, Berlin (1988), 91-95.

[20] G. Buttazzo, Semicontinuity, Relaxation and Integral Representation in the Calculus of Variations, Pitman Res. Notes Math. Ser. 207, Longman, Harlow, 1989.

[21] G. Buttazzo, Relaxed formulation for a class of shape optimization problems, Proceedings of Boundary Control and Boundary Variations, Sophia-Antipolis, October 15-17, 1990, Lecture Notes in Control and Inf. Sci. 178, Springer-Verlag, Berlin, (1992), 50-59.

[22] G. Buttazzo, Existence via relaxation for some domain optimization problems, Proceedings of Topology Design of Structures, Sesimbra 20-26 June 1992, NATO ASI Series E: Applied Sciences 227, Kluwer Academic Publishers, Dordrecht, (1993), 337-343.

[23] G. Buttazzo, Relaxed shape optimization problems with Dirichlet boundary conditions, Proceedings of Composite Media and Homogenization Theory II, Trieste 20 September - 1 October 1993, World Scientific, Singapore, (1995), 125-137.

[24] G. Buttazzo, A general theory of relaxed controls and application to shape optimization problems, Proceedings of Progress in Elliptic and Parabolic Partial Differential Equations, Capri September 19-23 1994, Pitman Res. Notes Math. Ser. 350, Longman, Harlow, (1996), 58-70.

[25] G. Buttazzo \& G. Dal Maso, Shape optimization for Dirichlet problems : relaxed solutions and optimality conditions, Bull. Amer. Math. Soc., 23, (1990), 531-535.

[26] G. Buttazzo \& G. Dal Maso, Shape optimization for Dirichlet problems : relaxed formulation and optimality conditions, Appl. Math. Optim. 23, (1991), 17-49.

[27] G. Buttazzo \& G. Dal Maso, An existence result for a class of shape optimization problems, Arch. Rational Mech. Anal., 122, (1993), 183-195.

[28] G. Buttazzo, G. Dal Maso, A. Garroni \& A. Malusa, On the relaxed formulation of Some Shape Optimization Problems, Adv. Math. Sci. Appl., (to appear).

[29] G. Buttazzo, V. Ferone \& B. Kawohl, Minimum problems over sets of concave functions and related questions, Math. Nachr., 173, (1995), 71-89. 
[30] G. Buttazzo \& B. Kawohl, On Newton's problem of minimal resistance, Math. Intelligencer, 15, (1993), 7-12.

[31] G. Buttazzo \& P. Guasoni, Shape optimization problems over classes of convex domains, Preprint Dipartimento di Matematica Università di Pisa, Pisa, 1997.

[32] G. Buttazzo \& A. Wagner, On the optimal shape of a rigid body supported by an elastic membrane, Preprint Dipartimento di Matematica Università di Pisa, Pisa, 1996.

[33] G. Buttazzo \& O.M. Zeine, Un problème d'optimisation de plaques, Modél. Math. Anal. Numér., 31, (1997), 167-184.

[34] E. CABIB, A relaxed control problem for two-phase conductors, Ann. Univ. Ferrara Sez. VII - Sc. Mat., 33, (1987), 207-218.

[35] E. Cabib \& G. Dal Maso, On a class of optimum problems in structural design, $J$. Optimization Theory Appl., 56, (1988), 39-65.

[36] J. CEA, Problems of shape optimal design, Proceedings of Optimization of Distributed Parameter Structures, Iowa City 1980, Sijthoff and Noordhoff, Rockville, (1981), 10051048.

[37] J. Cea \& K. Malanowski, An example of a max-min problem in partial differential equations, SIAM J. Control, 8, (1970), 305-316.

[38] D. Chenais, On the existence of a solution in a domain identification problem, J. Math. Anal. Appl, 52, (1975), 189-289.

[39] D. Chenais, Homeomorphisme entre ouverts voisins uniformement lipschitziens, $C . R$. Acad. Sci. Paris, A 283, (1976), 461-464.

[40] D. Chenais, Homeomorphisme entre ouverts lipschitziens, Ann. Mat. Pura Appl., 118, $(1980)$.

[41] M. Chipot \& G. Dal Maso, Relaxed shape optimization : the case of nonnegative data for the Dirichlet problem, Adv. Math. Sci. Appl. 1, (1992), 47-81.

[42] D. Cioranescu \& F. Murat, Un terme étrange venu d'ailleurs I, II, In Nonlinear Partial Differential Equations and Their Applications, Collège de France Seminar, Vol. II, 98-138, Vol III, 154-178, Pitman, Boston, 1982.

[43] G. Dal Maso, T-convergence and $\mu$-capacities, Ann. Scuola Norm. Sup. Pisa Cl. Sci., 14, (1987), 423-464.

[44] G. Dal Maso, An Introduction to T-convergence, Birkhäuser, Boston, 1993.

[45] G. Dal Maso \& A. Garroni, New results on the asymptotic behaviour of Dirichlet problems in perforated domains, Math. Mod. Meth. Appl. Sci., (to appear).

[46] G. Dal Maso \& A. Malusa, Approximation of relaxed Dirichlet problems by boundary value problems in perforated domains., Proc. Roy. Soc. Edinburgh, (to appear).

[47] G. Dal Maso \& U. Mosco, Wiener's criteria and energy decay for relaxed Dirichlet problems, Arch. Rational. Mech. Anal., 95, (1986), 345-387.

[48] G. Dal Maso \& U. Mosco, Wiener's criterion and T-convergence, Appl. Math. Optim., 15, (1987), 15-63.

[49] G. Dal Maso \& F. Murat, Asymptotic behaviour and correctors for Dirichlet problems in perforated domains with homogeneous monotone operators, Preprint SISSA, Trieste, 1996.

[50] S. Finzi Vita, Numerical shape optimization for relaxed Dirichlet problems, Math. Mod. Meth. Appl. Sci, 3, (1993), 19-34.

[51] J. Heinonen, T. Kilpelainen \& O. Martio, Nonlinear Potential Theory of Degenerate Elliptic Equations, Clarendon Press, 1993. 
[52] A. Henrot, Continuity with respect to the domain for the Laplacian: a survey, Control and Cybernetics, 23, (1994), 427-443.

[53] B. Kawohl, Remarks on some old and current eigenvalue problems, In Partial Differential Equations of Elliptic type, A. Alvino, E. Fabes, G. Talenti editors, Symposia Mathematica, vol. XXXV, Cambridge University Press, 1994.

[54] R.V. Kohn \& G. Strang, Optimal design and relaxation of variational problems, I, II, III, Comm. Pure Appl. Math., 39, (1986), 113-137, 139-182, 353-377.

[55] R.V. Kohn \& M. Vogelius, Relaxation of a variational method for impedance computed tomography, Comm. Pure Appl. Math., 40, (1987), 745-777.

[56] K.A. LuRie \& A.V. Cherkaev, G-closure of a set of anisotropically conductivity media in the two-dimensional case, J. Optimization Theory Appl, 42, (1984), 283-304.

[57] K.A. Lurie \& A.V. Cherkaev, G-closure of some particular sets of admissible material characteristics for the problem of bending of thin elastic plates, J. Optimization Theory Appl., 42, (1984), 305-316.

[58] F. Murat, Control in coefficients, Encyclopedia of systems and control, Pergamon Press, (1983), 808-812.

[59] F. Murat \& J. Simon, Etude de problèmes d'optimal design, Proceedings of Optimization Techniques. Modelling and Optimization in the Service of Man, Nice 1975, Lecture Notes in Computer Science 41, Springer-Verlag, Berlin, (1976), 54-62.

[60] F. Murat \& J. Simon, Sur le contrôle par un domaine géometrique, Preprint 76015, Univ. Paris VI, Paris, 1976.

[61] F. Murat \& L. Tartar, Optimality conditions and homogenization, Proceedings of Nonlinear variational problems, Isola d'Elba 1983, Res. Notes in Math. 127, Pitman, London, (1985), 1-8.

[62] F. Murat \& L. Tartar, Calcul des variations et homogénéisation, Proceedings of Les Méthodes de l'homogénéisation: Théorie et applications en physique, École d'Été d'Analyse Numérique C.E.A.-E.D.F.-INRIA, Bréau-sans-Nappe 1983, Collection de la direction des études et recherches d'électricité de France 57, Eyrolles, Paris, (1985), 319-369.

[63] O. Pironneau, Optimal Shape Design for Elliptic Systems, Springer-Verlag, Berlin, 1984.

[64] N. ShimakuRa, La première valeur propre du Laplacien pour le problème de Dirichlet, J. Math. Pures Appl, 62, (1983), 129-152.

[65] J. Sokolowski \& J.P. Zolesto, Introduction to Shape Optimization, SpringerVerlag, Berlin, 1992.

[66] V. Šverák, On optimal shape design, C. R. Acad. Sci. Paris, I-315, (1992), 545-549.

[67] V. Šverák, On optimal shape design, J. Math. Pures. Appl, 72, (1993), 537-551.

[68] L. Tartar, Estimations Fines des Coefficients Homogénéises, In Ennio De Giorgi Colloqium, Edited by P.Krée, Res. Notes in Math. 125, Pitman, London, (1985), 168-187.

[69] L. Tartar, Problèmes de contrôle des coefficients dans des équations aux dérivées partielles, In Control theory, numerical methods and computer systems modelling, Lecture Notes in Econom. and Math. Systems 107, Springer-Verlag, Berlin, (1975), 420-426.

[70] S.A. Wolf \& J.B. Keller, Range of the first two eigenvalues of the laplacian, Proc. R. Soc. Lond., A 447, (1994), 397-412. 